\title{
CARACTERÍSTICAS FÍSICAS DE LA MADERA DE PINO PROCEDENTE DE RALEOS EN EL NOROESTE DE ESPAÑA
}

\section{PHYSICAL PROPERTIES OF WOOD FROM THINNED PINES IN NORTHWEST SPAIN}

\author{
G. Riesco Muñoz ; J. Díaz González ${ }^{2}$
}

\begin{abstract}
RESUMEN
Se estudió la densidad, contracción, higroscopicidad y punto de saturación de la pared celular en la madera de ejemplares jóvenes de tres especies importantes en la producción maderera española:

Pinus pinaster, Pinus sylvestris y Pinus radiata. Se muestrearon 29 pinos procedentes de raleos efectuados en diferentes masas forestales de Galicia (noroeste de España). El material de ensayo fueron probetas de pequeñas dimensiones sin defectos. La madera de Pinus pinaster resultó semipesada y más inestable en dimensiones ante los cambios de humedad que las de Pinus radiata y Pinus sylvestris, que resultaron ligeras y relativamente estables, especialmente Pinus radiata. La densidad en Pinus pinaster fue similar a la obtenida en estudios anteriores sobre pies adultos. Pinus radiata y Pinus sylvestris presentaron madera más ligera que la de pies adultos. Las propiedades relativas a la estabilidad dimensional fueron similares en la madera joven analizada y en la madera de pies adultos, a excepción de la madera de Pinus radiata, que resultó más estable en la muestra que en la madera adulta. La variación entre zonas del fuste y entre individuos fue notable para las variables densidad y peso específico, aun en el caso de árboles muestreados en un mismo rodal y estación.
\end{abstract}

Palabras clave: Pinus, propiedades físicas de la madera, clara, raleo, Galicia.

\begin{abstract}
Wood density, shrinkage, hygroscopicity and fibre saturation point were studied in young trees corresponding to three important species in the spanish timber production (Pinus pinaster, Pinus sylvestris and Pinus radiata). Twenty-nine thinned pines from different stands of Galicia (northwestern Spain) were sampled. The test material was small size defect-free specimens. The wood of Pinus pinaster exhibited intermediate density and more instability in dimensions, due to environmental moisture changes, than wood of Pinus radiata and Pinus sylvestris. These woods were light and relatively stable, especially Pinus radiata. The density in Pinus pinaster was similar to that obtained in previous studies on adult trees. Pinus radiata and Pinus sylvestris showed lower density than adult trees. The shrinkage properties were similar in the analyzed young wood and in the wood of adult trees, excepting the wood sampled for Pinus radiata, more stable than adult wood. Even with a sample of trees from the same stand in a specific site, there were significant differences in wood density among positions in each tree and among trees.
\end{abstract}

Keywords: Pinus, physical properties of wood, thinning, Galicia.

\footnotetext{
${ }^{1}$ Doctor Ingeniero de Montes y Profesor Titular de Escuela Universitaria. Departamento de Ingeniería Agroforestal. Universidad de Santiago de Compostela. Lugo. España

${ }^{2}$ Ingeniero Técnico Forestal Escuela Politécnica Superior. Departamento de Ingeniería Agroforestal. Universidad de Santiago de Compostela. Lugo. España.

Autor para correspondencia: riescomu@lugo.usc.es

Recibido: 18.07.2007.Aceptado: 17.09. 2007.
} 


\section{INTRODUCCION}

La producción maderera en España alcanzó los 15.608.615 m³ con corteza en 2003, según las estadísticas más recientes (Ministerio de Agricultura, Pesca y Alimentación, 2007). La mayor parte de dicha producción (el $51 \%$ ) procede de Galicia, región del noroeste peninsular que, a pesar de sus altas producciones, solamente alberga el 7,9\% de la superficie forestal arbolada del país, según datos del Tercer Inventario Forestal Nacional (Ministerio de Medio Ambiente, 2007). La producción maderera de Galicia corresponde en un 52,4\% a cortas de coníferas, entre las que la especie Pinus pinaster es la más destacada, con $2.400 .467 \mathrm{~m}^{3}$ con corteza anuales, seguida de Pinus radiata, con $560.514 \mathrm{~m}^{3}$ anuales y por Pinus sylvestris, con $227.852 \mathrm{~m}^{3}$ anuales, según datos de 2003 (Ministerio de Agricultura, Pesca y Alimentación, 2007).

La especie Pinus pinaster se encuentra ampliamente extendida en Galicia mientras que las otras dos especies de pinos tienen una distribución más restringida: Pinus radiata se encuentra principalmente en el norte y Pinus sylvestris en la zona oriental de la región (Ministerio de Agricultura, Pesca y Alimentación, 2007).

Las tres especies proceden en Galicia de repoblaciones artificiales. En el caso de Pinus pinaster y Pinus radiata éstas se iniciaron en el siglo XIX y en el caso de Pinus sylvestris se iniciaron hace unos 50 años. Las especies Pinus pinaster y Pinus radiata son especies de crecimiento rápido que se tratan con turnos cortos (35-45 años para Pinus pinaster y 25-35 años para Pinus radiata) mientras que Pinus sylvestris es una especie de crecimiento lento que se trata con turnos largos (80-120 años). Por tanto, desde hace años se aprovechan masas maduras de Pinus pinaster y Pinus radiata en tanto que las masas de Pinus sylvestris son todavía masas jóvenes en las que solo se aprovechan fustes (troncos) de pequeño diámetro cuando se realizan raleos y otras cortas en una fase intermedia de desarrollo de las masas.

La mayor parte de la madera de pino producida en Galicia recibe la primera transformación en industrias radicadas en Galicia. El destino de la madera de pino es la industria de aserrado y chapa plana en el caso de las trozas de mayor diámetro y calidad. Las trozas delgadas (trozas con diámetro con corteza en punta delgada inferior a $15 \mathrm{~cm}$ ) y las trozas defectuosas se destinan a la industria de desintegración, para fabricar tableros de fibras y tableros de partículas. Estas trozas de peor calidad proceden de la parte superior de árboles adultos y también proceden de pies jóvenes obtenidos en raleos.

Los raleos en pinares son un tratamiento selvícola cada vez más frecuente en Galicia ya que suponen un aporte de madera adicional a una industria que presenta una fuerte demanda de materia prima. Es previsible que cada vez se aproveche más madera procedente de raleos por lo que deberían estudiarse las propiedades físicas de este material para conocer su aptitud tecnológica en la industria.

Este estudio pretende, en primer lugar, determinar qué diferencias se dan entre las tres especies de pino que crecen en Galicia en cuanto a las propiedades físicas de la madera de árboles jóvenes. Se comprobará, por tanto, si las tres clases de maderas son similares y, por tanto, serían "intercambiables" como materia prima en la industria. Por otra parte, la madera procedente de árboles maduros de las tres especies ya ha sido caracterizada en diversos estudios previos (e.g.: Casado et al., 2001; FernándezGolfín Seco, 1996; Fernández-Golfín Seco et al., 1997; Fonseca, 1989; Forest Products Laboratory, 1999; Gutiérrez Oliva y Plaza Pulgar, 1967; Gutiérrez Oliva et al., 1997; Hermoso et al., 2003; Remacha, 1987; Tuset y Durán, 1986; Vignote Peña y Jiménez Peris, 2000; Wagenführ y Scheiber, 1974; Wenger, 1984). Por ello, un segundo objetivo consiste en establecer las diferencias que puedan existir entre la madera de pinos jóvenes y la madera de pinos adultos en cuanto a las propiedades físicas de mayor interés en la industria. Por último, dado que en los raleos se aprovecha la madera de diferentes zonas del tronco, se analizará la variación a lo largo del fuste de las propiedades de la madera. 


\section{MATERIAL Y MÉTODOS}

La madera empleada en el estudio procede de ejemplares jóvenes de pino pinaster (Pinus pinaster Ait.), pino insigne (Pinus radiata D. Don) y pino silvestre (Pinus sylvestris L.) apeados en diferentes localizaciones de Galicia (España). Los datos generales de los árboles de la muestra se indican en la Tabla 1.

Tabla 1. Características de los 29 pinos muestreados. $n$ = número de árboles de la muestra.

\begin{tabular}{|c|c|c|c|c|c|c|}
\hline \multirow{2}{*}{ Especie } & \multicolumn{2}{|c|}{ localización } & \multirow{2}{*}{$\begin{array}{l}\text { altitud } \\
\text { (m) }\end{array}$} & \multirow{2}{*}{$n$} & \multirow{2}{*}{$\begin{array}{l}\text { edad } \\
\text { (años) }\end{array}$} & \multirow{2}{*}{$\begin{array}{c}\text { diámetro } \\
\text { normal } \\
(\mathrm{cm})\end{array}$} \\
\hline & $\begin{array}{l}\text { término } \\
\text { municipal }\end{array}$ & $\begin{array}{l}\text { coordenadas } \\
\text { geodésicas }\end{array}$ & & & & \\
\hline Pinus pinaster Ait. & Punxín & $42^{\circ} 21^{\prime} \mathrm{N} ; 8^{\circ} 01^{\prime} \mathrm{O}$ & 375 & 19 & $10-15$ & $5-15$ \\
\hline \multirow{6}{*}{ Pinus radiata D. Don } & Baralla & $42^{\circ} 53^{\prime} \mathrm{N} ; 7^{\circ} 14^{\prime} \mathrm{O}$ & 506 & 1 & \multirow{6}{*}{$15-25$} & \multirow{6}{*}{$19-24$} \\
\hline & Becerreá & $42^{\circ} 51^{\prime} \mathrm{N} ; 7^{\circ} 10^{\prime} \mathrm{O}$ & 648 & 1 & & \\
\hline & Corgo & $42^{\circ} 57^{\prime} \mathrm{N} ; 7^{\circ} 26^{\prime} \mathrm{O}$ & 443 & 1 & & \\
\hline & Guitiriz & $43^{\circ} 11^{\prime} \mathrm{N} ; 7^{\circ} 54^{\prime} \mathrm{O}$ & 450 & 1 & & \\
\hline & Láncara & $42^{\circ} 52^{\prime} \mathrm{N} ; 7^{\circ} 20^{\prime} \mathrm{O}$ & 460 & 1 & & \\
\hline & Monterroso & $42^{\circ} 47^{\prime} \mathrm{N} ; 7^{\circ} 50^{\prime} \mathrm{O}$ & 489 & 1 & & \\
\hline Pinus sylvestris $\mathrm{L}$. & Fonsagrada & $43^{\circ} 06^{\prime} \mathrm{N} ; 7^{\circ} 05^{\prime} \mathrm{O}$ & 900 & 4 & $24-28$ & $20-35$ \\
\hline
\end{tabular}

El número de pies muestreado fue aproximadamente proporcional a la extensión de cada especie en el territorio de Galicia. Los ejemplares de Pinus pinaster se obtuvieron en un rodal con estructura de masa regular procedente de regeneración natural después de incendio en las montañas del sur de Galicia. Los ejemplares de Pinus radiata procedían de diferentes repoblaciones del interior de la región en tanto que Pinus sylvestris se muestreó en una repoblación de las montañas orientales. De cada árbol se obtuvieron discos o rodajas de unos $10 \mathrm{~cm}$ de espesor y de la sección transversal completa del tronco a dos alturas en el fuste (en la base y a $5 \mathrm{~m}$ de altura). Con ello se pretendía abarcar la variación de propiedades de la madera en la zona del fuste de mayor valor comercial, que es la que corresponde a las dos primeras trozas: de 0 a 2,5 m y de 2,5 a $5 \mathrm{~m}$. Para la especie Pinus radiata no se dispuso de todas las rodajas a alturas fijas ya que la abundante nudosidad de los fustes en esta especie impedía tomar rodajas a las alturas previstas. Las rodajas fueron catalogadas y labradas para obtener las probetas prismáticas de pequeñas dimensiones y libres de defectos que habrían de ser el material de ensayo en laboratorio. Se seleccionaron probetas de pequeñas dimensiones sin defectos ya que de esta forma es posible efectuar comparaciones entre especies y procedencias. Se rechazaron las probetas con las aristas no paralelas a las direcciones principales de la madera y también fueron rechazadas las probetas con anillos muy curvos y próximos a la médula, a fin de excluir del análisis la madera juvenil. El número de probetas válidas elegidas en cada árbol fue variable. En total fueron ensayadas 319 probetas libres de defectos ( 241 probetas de 4 x 2 x $2 \mathrm{~cm}$ de dimensiones nominales y 78 probetas de 4 x 4 x $2 \mathrm{~cm}$ de dimensiones nominales). 
Sobre dichas probetas se analizaron las propiedades gravimétricas (peso específico, densidad) y la estabilidad dimensional de la madera frente a los cambios de humedad (contracciones, coeficientes de contracción y otros). La relación de propiedades físicas evaluadas aparece en la Tabla 2. El procedimiento de ensayo aplicado en la muestra es el que aparece en la normativa española UNE y europea EN relativa a la determinación e interpretación de propiedades físicas (AENOR, 1977a, 1977b, 1977c, 1978a, 1978b).

Para determinar las propiedades físicas fue necesario medir las tres dimensiones principales de cada probeta a tres niveles diferentes de humedad de la madera: a saturación de humedad, a una humedad de equilibrio higroscópico en torno al $12 \%$ y en estado anhidro. Asimismo, fue necesario obtener el peso de cada probeta a la humedad de equilibrio higroscópico y en estado anhidro.

Para medir las probetas de ensayo en condiciones de saturación de humedad se mantuvieron sumergidas en agua a temperatura ambiente durante 96 horas. Posteriormente, para medir las probetas a una humedad de equilibrio higroscópico de la madera próxima al $12 \%$, se homogeneizaron en la atmósfera del laboratorio hasta alcanzar dicha humedad. Por último, para llevar las probetas de ensayo al estado anhidro se mantuvieron en estufa con circulación de aire a $103{ }^{\circ} \mathrm{C}$ durante 48 horas y se realizó una segunda pesada a las 12 horas, para comprobar que la desecación había sido completa. Antes de proceder a la medición de las probetas desecadas, se efectuó el enfriado de las mismas en desecador de vidrio, con gel de sílice como agente desecante, para evitar que absorbieran humedad.

El volumen de cada probeta se obtuvo multiplicando sus tres dimensiones principales, considerando cada probeta como un prisma perfecto. Para las mediciones se empleó micrómetro y calibre con precisión de $0,01 \mathrm{~mm}$. Para las pesadas se empleó balanza con precisión de $0,01 \mathrm{~g}$.

En cada probeta se registró la anchura de anillos contando el número de anillos sobre un segmento radial lo más largo posible en cada testa de la probeta. La anchura de anillos en cada testa se obtuvo como cociente entre la longitud del segmento y el número de anillos contabilizado. Como anchura de anillos de la probeta se tomó la media de las dos testas.

Para detectar la presencia de duramen las probetas ya ensayadas fueron sumergidas en una solución acuosa al $1 \%$ de anaranjado de metilo, que tiñe de amarillo las zonas de albura y de rojo el duramen, debido a la mayor acidez de éste.

La humedad de equilibrio higroscópico se calculó por diferencia de pesadas entre estado húmedo y estado anhidro, dividiendo por el peso anhidro. El peso específico a la humedad de referencia del $12 \%$ $\left(\rho_{12}\right)$ fue obtenido como cociente entre peso y volumen, ambos a dicha humedad de referencia. El peso específico anhidro fue obtenido como cociente entre peso y volumen anhidro $\left(\rho_{0}\right)$. La densidad básica se calculó como cociente entre el peso anhidro de la probeta y su volumen a saturación de humedad $\left(d_{b}\right)$.

La contracción volumétrica $\left(C_{v}\right)$ y la contracción en cada dirección principal $\left(C_{t}, C_{r}\right.$ y $\left.C_{t}\right)$ se calcularon como la variación de dimensión entre el estado de saturación de humedad y el estado anhidro, expresada en porcentaje de la dimensión en estado anhidro. El coeficiente de contracción volumétrica $(v)$ y el coeficiente de contracción lineal en cada dirección principal (lineal, radial y tangencial) se calcularon como la variación de dimensión entre el estado de equilibrio higroscópico y el estado anhidro, expresada en porcentaje de la dimensión en estado anhidro y por grado de humedad.

Se analizó el comportamiento de la madera a la contracción (y no a hinchazón) por ser el fenómeno habitual en la madera recién aserrada y por existir normativa española de ensayo al respecto. Para el lector más habituado a manejar las propiedades físicas de la madera en base húmeda se incluyen 
también los resultados sobre base húmeda para la humedad y para las cuatro propiedades de contracción (Tabla 2).

El punto de saturación de la pared celular se calculó como la relación entre la contracción volumétrica y su coeficiente. La higroscopicidad se obtuvo mediante la expresión:

$$
h i=0,01(1-v) \rho_{12}
$$

donde $h i$ es la higroscopicidad en $\mathrm{kg} / \mathrm{m}^{3}$

$v$ es el coeficiente de contracción volumétrica en porcentaje

$\rho_{12}$ es el peso específico aparente en $\mathrm{kg} / \mathrm{m}^{3}$ al $12 \%$ de humedad de la madera (AENOR, 1977b).

Se calcularon las constantes de Koelher (citado por Remacha et al., 1993), que establecen para cualquier clase de madera una relación de proporcionalidad entre contracción (en porcentaje) y peso específico anhidro (en $\mathrm{g} / \mathrm{cm}^{3}$ ) en un rango de humedades de equilibrio higroscópico entre el 5 y el 25 $\%$.

En general, la interpretación de los resultados de los ensayos se basó en la norma UNE (AENOR, 1978b). Sin embargo, para algunas variables no existe norma de interpretación de resultados por lo que se acudió, en tales casos, a criterios de interpretación alternativos (Peraza Oramas, 1988') y a la comparación con los resultados obtenidos por otros autores.

Para la determinación de diferencias estadísticamente significativas entre medias de las variables físicas analizadas se utilizó la prueba no paramétrica de Kruskal-Wallis. 


\section{RESULTADOS Y DISCUSION}

Los principales estadísticos descriptivos de las variables analizadas se encuentran en la Tabla 2.

Tabla 2. Estadísticos descriptivos de las variables físicas analizadas en la tres especies de maderas. Valor medio y, entre paréntesis, coeficiente de variación en porcentaje.

\begin{tabular}{|c|c|c|c|}
\hline Variable & Pinus pinaster & Pinus radiata & Pinus sylvestris \\
\hline anchura de anillos (mm) & $7,0(27,9)$ & $4,2(21,1)$ & $4,8(28,0)$ \\
\hline humedad (\%) & $9,8(4,9)$ & $12,4(13,5)$ & $12,3(8,9)$ \\
\hline peso específico al $12 \%\left(\mathrm{~kg} / \mathrm{m}^{3}\right)$ & $499(12,6)$ & $413(7,0)$ & $470(10,6)$ \\
\hline peso específico anhidro $\left(\mathrm{kg} / \mathrm{m}^{3}\right)$ & $466(12,9)$ & $381(6,8)$ & $440(10,8)$ \\
\hline densidad básica $\left(\mathrm{kg} / \mathrm{m}^{3}\right)$ & $408(11,7)$ & $345(6,5)$ & $391(11,0)$ \\
\hline contracción volumétrica (\%) & $13,8(19,2)$ & $10,7(17,4)$ & $12,3(12,4)$ \\
\hline coeficiente de contracción volumétrica (\%) & $0,39(16,2)$ & $0,27(37,5)$ & $0,40(12,2)$ \\
\hline punto de saturación de la pared celular (\%) & $35(16,7)$ & $51(82,1)$ & $31(9,2)$ \\
\hline higroscopicidad $\left(\mathrm{kg} / \mathrm{m}^{3}\right)$ & $3,0(13,7)$ & $3,0(17,0)$ & $2,8(11,0)$ \\
\hline constante de Koelher volumétrica & $30(14,3)$ & $28(17,0)$ & $28(14,4)$ \\
\hline contracción longitudinal (\%) & $1,0(83,9)$ & $0,7(60,3)$ & $0,7(86,2)$ \\
\hline coeficiente de contracción longitudinal (\%) & $0,04(87,0)$ & $0,03(68,9)$ & $0,03(73,8)$ \\
\hline constante de Koelher longitudinal & $2(81,3)$ & $2(64,2)$ & $2(77,4)$ \\
\hline contracción radial (\%) & $5,0(23,7)$ & $3,9(14,3)$ & $4,2(25,5)$ \\
\hline coeficiente de contracción radial (\%) & $0,15(23,1)$ & $0,08(43,9)$ & $0,15(25,1)$ \\
\hline constante de Koelher radial & $11(20,4)$ & $10(14,6)$ & $10(26,5)$ \\
\hline contracción tangencial (\%) & $7,2(20,2)$ & $5,7(12,6)$ & $7,1(18,7)$ \\
\hline coeficiente de contracción tangencial (\%) & $0,20(20,1)$ & $0,16(43,3)$ & $0,22(16,3)$ \\
\hline constante de Koelher tangencial & $16(16,9)$ & $15(13,1)$ & $16(20,1)$ \\
\hline humedad en base húmeda $(\%)$ & $8,9(4,4)$ & $11,0(12,2)$ & $10,9(8,1)$ \\
\hline contracción volumétrica en base húm. (\%) & $12,1(17,0)$ & $9,6(15,5)$ & $10,9(11,1)$ \\
\hline contracción longitudinal en base húm. (\%) & $1,0(82,1)$ & $0,7(59,8)$ & $0,6(85,0)$ \\
\hline contracción radial en base húmeda $(\%)$ & $4,8(22,5)$ & $3,7(13,8)$ & $4,0(24,7)$ \\
\hline contracción tangencial en base húmeda (\%) & $6,7(18,9)$ & $5,4(12,0)$ & $6,6(17,2)$ \\
\hline
\end{tabular}

En Galicia, las especies Pinus pinaster y Pinus radiata son de crecimiento rápido en tanto que Pinus sylvestris es una especie de crecimiento lento. La anchura media de anillos de crecimiento es algo inferior en Pinus radiata, circunstancia no esperable al tratarse de una especie de crecimiento más rápido que Pinus sylvestris. Asimismo, la anchura media de anillos es algo superior en Pinus pinaster, lo cual puede deberse a que ésta es la especie más joven de entre las muestreadas (Tabla 1). En todo caso, al ser escasa la diferencia en cuanto a anchura de anillos entre especies, ésta no se considerará una variable explicativa de las diferencias que se encuentren entre especies en la muestra analizada.

La humedad de equilibrio higroscópico en las probetas de la muestra fue, en promedio, muy próxima a la humedad de referencia del 12,0\%. En el caso de Pinus pinaster la humedad de equilibrio alcanzada por la muestra difiere ligeramente de la referencia, aunque se trata de valores sobre los que es posible aplicar una corrección sobre el peso específico húmedo para expresarlo al 12 \% (AENOR, 1977a). 
Para todas las variables gravimétricas y de estabilidad dimensional se han observado diferencias estadísticamente significativas o altamente significativas entre especies, salvo para el coeficiente de contracción longitudinal (Tabla 3). Esta excepción puede atribuirse a la muy elevada dispersión de resultados que presenta esta variable en las tres especies (Tabla 2), dispersión que probablemente impide detectar diferencias significativas.

Tabla 3. Estadísticos de contraste de diferencias significativas entre medias para las variables analizadas, empleando la especie como variable de agrupación. Prueba de Kruskal-Wallis.

\begin{tabular}{|l|c|c|c|}
\hline \multicolumn{1}{|c|}{ Variable } & Chi-cuadrado & $\begin{array}{c}\text { Grados de } \\
\text { libertad }\end{array}$ & $\begin{array}{c}\text { Significación } \\
\text { asintótica }\end{array}$ \\
\hline peso específico al 12\% $\left(\mathrm{kg} / \mathrm{m}^{3}\right)$ & 123,201 & 2 & 0,0000 \\
\hline peso específico anhidro $\left(\mathrm{kg} / \mathrm{m}^{3}\right)$ & 132,486 & 2 & 0,0000 \\
\hline densidad básica $\left(\mathrm{kg} / \mathrm{m}^{3}\right)$ & 120,309 & 2 & 0,0000 \\
\hline contracción volumétrica (\%) & 87,060 & 2 & 0,0000 \\
\hline coeficiente de contracción volumétrica (\%) & 93,372 & 2 & 0,0000 \\
\hline punto de saturación de la pared celular (\%) & 32,127 & 2 & 0,0000 \\
\hline higroscopicidad (kg/m $\left.{ }^{3}\right)$ & 13,254 & 2 & 0,0013 \\
\hline constante de Koelher volumétrica & 18,743 & 2 & 0,0001 \\
\hline contracción longitudinal (\%) & 15,989 & 2 & 0,0003 \\
\hline coeficiente de contracción longitudinal (\%) & 0,106 & 2 & 0,9486 \\
\hline constante de Koelher longitudinal & 11,508 & 2 & 0,0032 \\
\hline contracción radial (\%) & 40,217 & 2 & 0,0000 \\
\hline coeficiente de contracción radial (\%) & 60,754 & 2 & 0,0000 \\
\hline constante de Koelher radial & 9,566 & 2 & 0,0084 \\
\hline contracción tangencial (\%) & 34,475 & 2 & 0,0000 \\
\hline coeficiente de contracción tangencial $(\%)$ & 16,294 & 2 & 0,0003 \\
\hline constante de Koelher tangencial & 9,187 & 2 & 0,0101 \\
\hline
\end{tabular}

La existencia de diferencias estadísticamente significativas se encuentra asociada a diferencias apreciables entre las tres especies, salvo en el caso de la higroscopicidad y las cuatro constantes de Koelher, poco diferentes entre especies.

Para cada especie se encontraron también diferencias significativas entre individuos para las propiedades de densidad y peso específico. Por el contrario, no siempre se encontraron diferencias entre individuos para las propiedades relacionadas con la estabilidad dimensional. Se observó que la madera de Pinus radiata es más ligera que la de Pinus pinaster y Pinus sylvestris. En concreto, por unidad de volumen saturado de humedad, Pinus sylvestris presentaría un $13 \%$ más de materia seca que Pinus radiata y Pinus pinaster presentaría un $18 \%$ más que Pinus radiata.

Por la importancia del peso específico y la densidad como indicadores de calidad en la caracterización de la madera de coníferas se incluye el diagrama de cajas correspondiente al peso específico al $12 \%$ de humedad (Figura 1). Según la norma de interpretación UNE (AENOR, 1978b), el peso específico medio obtenido en la muestra de Pinus radiata y Pinus sylvestris informa de dos especies de maderas ligeras mientras que la madera de Pinus pinaster entraría en el grupo de las semipesadas. 


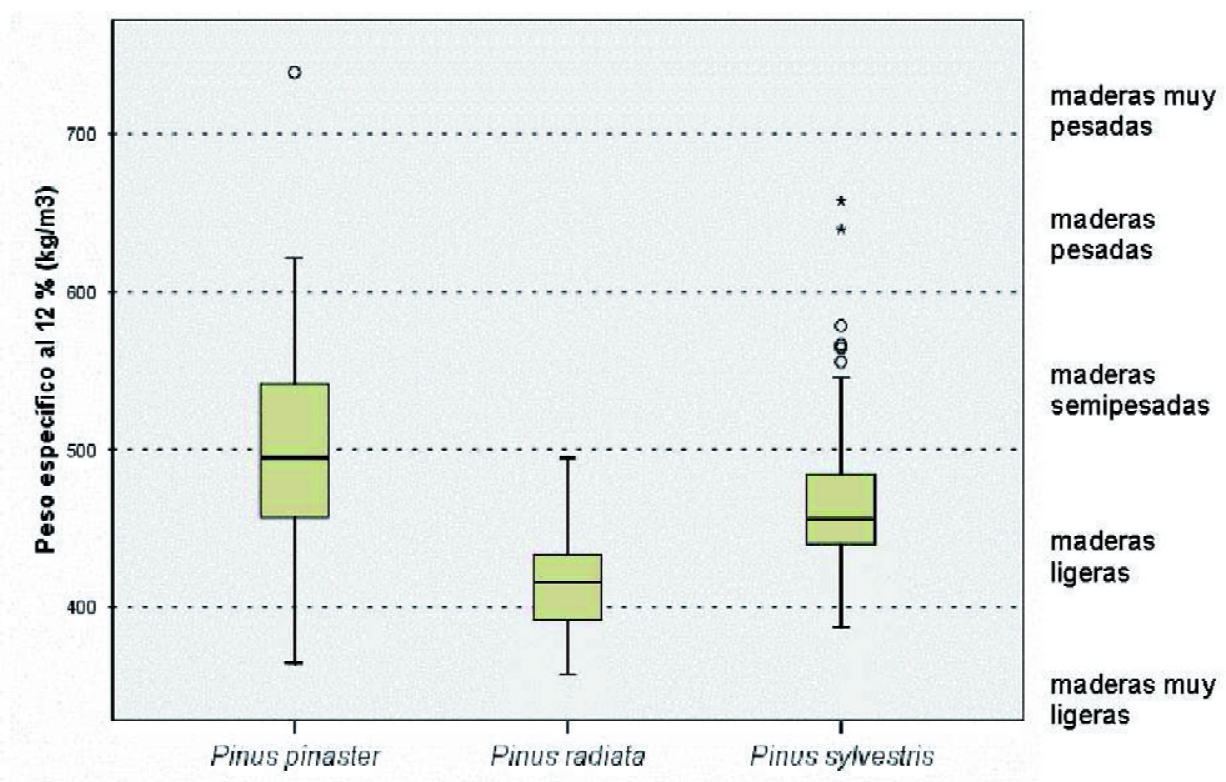

Figura 1. Distribución del peso específico de la madera de pino procedente de raleos en las tres especies estudiadas (Galicia, España).

La baja densidad en la madera de Pinus radiata permite suponer que va a tratarse de una madera con un comportamiento estable ante las variaciones de humedad (Kininmonth y Whitehouse, 1991). En efecto, el examen de las variables de contracción volumétrica, radial y tangencial muestra que la madera de Pinus radiata es más estable dimensionalmente frente a los cambios de humedad que las otras dos especies.

La contracción volumétrica es de tipo medio en las tres especies por lo que se producirán fendas de tipo medio durante el secado. La madera de Pinus radiata es poco nerviosa y, por tanto, es válida para carpintería, ebanistería y tornería. Por el contrario, la madera de Pinus pinaster y Pinus sylvestris es medianamente nerviosa por lo que su aplicación aconsejable sería como madera de construcción.

El punto de saturación de la pared celular es normal en Pinus pinaster y Pinus sylvestris aunque es elevado en Pinus radiata. En efecto, el punto de saturación se ha estimado como cociente entre la contracción volumétrica y su coeficiente por lo que el alto punto de saturación de la pared celular obtenido en Pinus radiata es atribuible a que esta madera presenta un coeficiente de contracción volumétrica claramente inferior al obtenido en las otras dos especies en tanto que la contracción volumétrica total no difiere apreciablemente entre especies. La higroscopicidad obtenida es normal según la norma de interpretación UNE (AENOR, 1978b).

La contracción radial y la tangencial son débiles en Pinus radiata y moderadas, tendiendo a débiles, en Pinus pinaster y Pinus sylvestris, según el criterio de interpretación de Peraza Oramas (1988). La contracción longitudinal es muy inferior a la contracción en dirección radial y tangencial. Esto permite suponer que la muestra no contiene madera juvenil en cantidad apreciable (la madera juvenil fue casi excluida del estudio al rechazar para ensayos las probetas que presentaban médula). En promedio, la contracción en dirección longitudinal es de 5 a 6 veces inferior a la contracción en dirección radial y de 
7 a 10 veces inferior a la contracción en dirección tangencial. De igual forma, el coeficiente de contracción longitudinal es muy inferior al coeficiente en cualquiera de las otras dos direcciones principales de la madera.

Como ya se ha dicho, la mayor estabilidad de la madera de Pinus radiata puede atribuirse a su menor peso específico. Para comparar las propiedades de contracción entre especies en las que los pesos específicos difieren, se pueden emplear las constantes de Koelher. Éstas representan el cociente entre una variable de contracción y el peso específico. En la muestra, los valores obtenidos para las constantes de Koelher se ajustan solo en parte a los valores teóricos propuestos por dicho autor $\left(C_{v} / \rho_{0}=28,0 ; C_{l} / \rho_{0}=1,5 ; C_{r} / \rho_{0}=9,5 ; C_{t} / \rho_{0}=17,0\right)$. En concreto, la madera de Pinus radiata y Pinus sylvestris presenta valores similares a los teóricos. Es decir, su estabilidad dimensional es la que cabe esperar para su peso específico. Por el contrario, Pinus pinaster es algo más inestable de lo que cabría esperar en función de su peso específico.

Finalmente, los resultados del estudio, obtenidos sobre madera de pies jóvenes procedentes de raleos, se han comparado con los resultados recopilados en diversas fuentes bibliográficas para madera procedente de pies adultos. En el caso de Pinus pinaster, el peso específico, la densidad básica y la mayoría de las variables que describen la estabilidad dimensional de la madera presentan valores similares a los que se citan para la madera de pies adultos. Algunas propiedades (el punto de saturación de la pared celular, la contracción radial y las variables de contracción longitudinal) presentan valores superiores a los correspondientes a madera adulta (Carvalho, 1994; Casado et al., 2001; FernándezGolfín Seco y Díez Barra, 1995; Fonseca, 1989; Gutiérrez Oliva y Plaza Pulgar, 1967; Ortiz y Martínez, 1991; Penetra, 1991; Prades et al., 2001; Remacha, 1987; Sánchez Plaza, 1969; Tuset y Durán, 1986; Vignote Peña y Jiménez Peris, 2000).

En el caso de Pinus radiata la madera analizada es bastante más ligera y estable que la madera procedente de pies adultos, aunque es más higroscópica y presenta mayor contracción longitudinal que la madera de madurez (Fernández-Golfín Seco, 1996; Fernández-Golfín Seco y Díez Barra, 1995; Forest Products Laboratory, 1999; Guindeo Casasœs y García Esteban, 1988; Gutiérrez Oliva y Plaza Pulgar, 1967; López de Roma, 1991; Remacha et al., 1993; Sánchez Plaza, 1969; Tuset y Durán, 1986; Vignote Peña y Jiménez Peris, 2000; Wagenführ y Scheiber, 1974).

En el caso de Pinus sylvestris, el peso específico, la densidad básica y la higroscopicidad toman valores inferiores a los que se citan para la madera de pies adultos. Las demás propiedades toman valores similares en pies jóvenes y en pies maduros, a excepción de las contracciones longitudinal y radial, que son superiores en los pies jóvenes analizados (AITIM, 1997; Fernández Rodríguez, 2002; Fernández-Golfín Seco y Díez Barra, 1994; Fernández-Golfín Seco et al., 1997; Guindeo Casasús y García Esteban, 1988; Gutiérrez Oliva y Plaza Pulgar, 1967; Gutiérrez Oliva et al., 1997; Hermoso et al., 2003; López de Roma, 1991; Nájera y Angulo y Peraza Oramas, 1960; Nutsch, 1992; Peraza Oramas y Guindeo Casasús, 1976; Sánchez Plaza, 1969; Tuset y Durán, 1986; Vignote Peña y Jiménez Peris, 2000; Wagenführ y Scheiber, 1974; Wenger, 1984).

Finalmente, se comparó el peso específico al $12 \%$ de humedad de la madera en las dos localizaciones del fuste de donde se tomaron muestras (en la base del fuste y a $5 \mathrm{~m}$ de altura). En los ejemplares de Pinus pinaster se encontró que el peso específico decrece con la altura ya que en la base el peso específico es un $19 \%$ mayor que a $5 \mathrm{~m}$ de altura, variando ese porcentaje entre árboles (desde el 0 al 33 $\%)$. En Pinus radiata no se encontró una tendencia clara. En Pinus sylvestris se encontró que el peso específico decrece levemente con la altura (un $5 \%$ en $5 \mathrm{~m}$ ) en dos de los pies analizados ya que en los otros dos el tamaño de muestra era insuficiente como para obtener pautas de variación del peso específico con la altura. A la vista de los resultados, en Pinus pinaster parece aconsejable aprovechar principalmente la primera troza (de 0 a $2,5 \mathrm{~m}$ de altura) tanto por su dimensión como por la mayor proporción que 
presenta de materia leñosa. En Pinus sylvestris, el aprovechamiento comercial de una o más trozas en madera de raleos vendrá determinado fundamentalmente por el diámetro de las piezas ya que no se aprecian diferencias notables en cuanto a densidad de la madera. Se asume aquí que la mayor parte de la madera de raleos de estas especies va a destinarse a trituración. No obstante, teniendo en cuenta la estabilidad dimensional, similar a la de la madera adulta, la madera de raleos podría emplearse en la industria de aserrado, siempre que las dimensiones de las trozas fueran suficientes.

\section{CONCLUSIONES}

Cabría esperar que la madera de las especies de crecimiento rápido (Pinus pinaster y Pinus radiata) presentaría características diferentes a la madera de la especie de crecimiento lento (Pinus sylvestris). Sin embargo, Pinus radiata es la especie que ha mostrado características claramente diferentes de las otras dos. En concreto, ha resultado ser una madera significativamente más ligera y más estable dimensionalmente frente a los cambios de humedad. Esto permite sugerir que el uso más indicado para la madera joven de Pinus radiata no es la industria de trituración ya que en ésta se precisa una elevada proporción de materia seca, que la especie no aporta, mientras que la estabilidad dimensional de la especie es una característica poco relevante en la industria de tableros. Por el contrario, cuando la dimensión de las trozas lo permita, Pinus radiata sería una especie indicada en la industria de aserrado para la fabricación de elementos de madera maciza destinados a carpintería y ebanistería. Por otra parte, Pinus pinaster y Pinus sylvestris, sin ser plenamente sustituibles entre sí, ya que presentan apreciables diferencias en la mayoría de las propiedades, tienen un destino más adecuado en la industria del tablero debido a su mayor peso específico y peor comportamiento dimensional ante los cambios de humedad.

En las tres especies analizadas, la madera de pies jóvenes presenta densidades y contracciones similares o inferiores a los valores propios de la madera pies adultos. Sin embargo, en las tres especies la contracción longitudinal es muy superior a los escasos resultados que cita la bibliografía consultada. (Cabría esperar valores más altos aún de contracción longitudinal si se hubiera incluido en el estudio la madera juvenil, muy inestable en dirección axial). Por tanto, si se emplea la madera de pinos jóvenes como madera maciza debe considerarse que su buen comportamiento en cuanto a estabilidad dimensional va unido a una elevada contracción en la dirección de la fibra. Esto puede considerarse como un distintivo de la madera que aún no ha llegado a la madurez.

El peso específico al $12 \%$ de humedad varía significativamente a lo largo del fuste, con una variación que depende del individuo y la especie. Por ello, en el aprovechamiento industrial del fuste debe considerarse las propiedades de la madera además de la dimensión de las trozas.

\section{BIBLIOGRAFIA}

AENOR. 1977a. Norma UNE 56531:1977. Determinación del peso específico. AENOR, Madrid.

AENOR.1977b. Norma UNE 56532:1977. Determinación de la higroscopicidad. AENOR, Madrid.

AENOR. 1977c. Norma UNE 56533:1977. Determinación de las contracciones lineal y volumétrica. AENOR, Madrid.

AENOR. 1978a. Norma UNE 56528:1978. Preparación de probetas para ensayos. AENOR, Madrid. 
AENOR. 1978b. Norma UNE 56540:1978. Interpretación de los resultados de los ensayos. AENOR, Madrid.

AITIM. 1997. Especies de maderas para carpintería, construcción y mobiliario. Asociación de Investigación Técnica de la Madera, Madrid.

Carvalho, A. 1994. Potencialidades da produçao pinícola nacional. Intensificaçao do crescimento e qualidade absoluta da madeira. Actas del III Congresso Florestal Nacional, vol. 2, 328-336.

Casado, M.; García, M.; Acuña, L. 2001. Caracterización físico-mecánica del Pinus pinaster Ait. procedencia Sierra de Oña (Burgos), influencia del factor rectitud. Actas del III Congreso Forestal Español, vol. 5, 545-553.

Fernández Rodríguez, C. 2002. Análisis comparativo de la densidad de la madera mediante la utilización del resistógrafo en Pinus sylvestris L. y Picea abies Karst. Proyecto Fin de Carrera, Universidad de Santiago de Compostela, Lugo.

Fernández-Golfín Seco, J.I. 1996. Manual de usuario pino insignis del País Vasco. Centro Técnico de la Madera, Vitoria.

Fernández-Golfín Seco, J.I.; Díez Barra, M.R. 1994. Influencia de la anchura del anillo de crecimiento en la densidad y otras propiedades físico-mecánicas de la madera estructural de diversas especies. Investigación Agraria. Sistemas y Recursos Forestales 3(2): 211-219.

Fernández-Golfín Seco, J.I.; Díez Barra, M. R. 1995. La anchura del anillo de crecimiento. Predictor de la calidad de la madera aserrada para uso estructural. Boletín de Información Técnica AITIM 173, 59-62.

Fernández-Golfín Seco, J.I.; Díez Barra, M.R.; Gutiérrez Oliva, A. 1997. Caracterización mecánica de la madera aserrada de pino silvestre de los Sistemas Central e Ibérico mediante probetas de tamaño estructural. Investigación Agraria. Sistemas y Recursos Forestales 6 (1- 2): 183-214.

Fonseca, F.M.A. 1989. Variaçao na madeira de Pinus pinaster Ait. Universidade de Trás-Os-Montes e Alto Douro, Vila Real.

Forest Products Laboratory. 1999. Wood Handbook. United States Department of Agriculture, Madison.

Guindeo Casasús, A.; García Esteban, L. 1988. Anatomía e identificación de las maderas de coníferas españolas. AITIM, Madrid.

Gutiérrez Oliva, A.; Plaza Pulgar, F. 1967. Características físico-mecánicas de las maderas españolas. IFIE, Madrid.

Gutiérrez Oliva, A.; Baonza Merino, M.V.; Fernández-Golfín Seco, J.I. 1997. Variaciones de la densidad de la madera de pino silvestre de los Sistemas Central e Ibérico. Actas del I Congreso Forestal Hispano-Luso IRATI 97, vol. 5, 229-234.

Hermoso, E.; Fernández-Golfín J.I.; Díez, M.R. 2003. Mechanical characterization of timber according to European standards from Spanish provenances of Scots Pine. Investigación Agraria. Sistemas y Recursos Forestales 12(3): 103-110. 
Kininmonth, J.A.; Whitehouse, L.J. 1991. Properties and uses of New Zealand Radiata Pine (vol. I, Wood properties). New Zealand Ministry of Forestry, Forest Research Institute, Rotorua.

López de Roma, A. 1991. Propiedades y tecnología de la madera de pino radiata del País Vasco. Monografía n. ${ }^{\circ}$ 80. INIA-MAPA, Madrid.

Nájera y Angulo, F.; Peraza Oramas, C. 1960. Características fisico-mecánicas del pino silvestre en España. Anales del Instituto Forestal de Investigaciones y Experiencias 5: 83-107.

Nutsch, W. 1992. Tecnología de la madera y del mueble. Reverté S. A., Barcelona.

Ortiz, J.; Martínez, J.J. 1991. Características mecánicas de la madera de pino gallego obtenidas a partir de ensayos con piezas de tamaño estructural. Boletín de Información Técnica AITIM 150: 95101.

Penetra, J.L. 1991. Variaçao nas componentes da densidade na madeira de Pinus pinaster Ait. Série Técnica-Científica, 12. Universidade de Trás-Os-Montes e Alto Douro, Vila Real.

Peraza Oramas, C.; Guindeo Casasús, A. 1976. Tecnología de la madera. vol. 4. La madera como materia prima. AITIM, Madrid.

Prades, C.; Montero, A.; Rubio, J. 2001. Características físico-mecánicas de la madera de Pinus pinaster Ait., procedente de los montes del Marquesado (provincia de Granada), Actas del III Congreso Forestal Español, vol. 5, 665-670.

Remacha, A. 1987. Características del pino gallego y sus aplicaciones. AITIM, Madrid.

Remacha, A.; Chozas, A.; Álvarez, H.; Ortiz, J. 1993. Curso de secado de maderas Uruguay. Universidad Politécnica de Madrid, Madrid.

Sánchez Plaza, A. 1969. Los pinos y abetos españoles. Utilización racional de su madera. Montes 148: 333-337.

Tuset, R.; Durán, F. 1986. Manual de maderas comerciales, equipos y procesos de utilización. Hemisferio Sur, Montevideo.

Vignote Peña, S.; Jiménez Peris, F.J. 2000.“Tecnología de la madera. MAPA-Mundiprensa, Madrid.

Wagenführ, R.; Scheiber, C. 1974. Holzatlas. Fachbuchverlag, Leipzig.

Wenger, K.F. 1984. Forestry Handbook. The Society of American Foresters ( $2^{\mathrm{a}}$ ed.). John Wiley \& Sons, New York. 